\title{
La capacidad de los pacientes para tomar decisiones: una tarea todavía pendiente
}

RESUMEN: La evaluación de la capacidad constituye un reto abierto para el modelo de relación clínica basado en la idea de consentimiento informado.

PALABRAS CLAVE: Capacidad, bioética, consentimiento informado, psiquiatría Legal.
SUMMARY: The evaluation of the capacity constitutes a challenge for the model of clinical relation based on the idea of informed consent.

KEY WORDS: Capacity, bioethics, informed consent, legal psychiatry.

Puede decirse que la evaluación de la capacidad es una de las cuestiones clave todavía no resueltas por la teoría general del consentimiento informado. Y ello a pesar de que en EE.UU. comenzó a ser motivo de preocupación hace ya casi treinta años, desde los mismos inicios de la era moderna del consentimiento informado en la práctica clínica, inaugurada por el caso Canterbury v Spence en 1972 (1).

Como es bien sabido, el consentimiento informado no es sino un modelo de relación clínica fruto del resultado de la introducción de la idea de autonomía psicológica y moral de las personas en el modelo clásico de relación médico-paciente, basado hasta entonces, exclusivamente, en la idea de beneficencia paternalista (2). El ejercicio de la autonomía de las personas, de los pacientes, exige que se cumplan al menos tres condiciones: 1. Actuar voluntariamente, es decir libre de coacciones externas 2 . Tener información suficiente sobre la decisión que va a tomar, es decir, sobre el objetivo de la decisión, sus riesgos, beneficios y alternativas posibles. 3. Tener capacidad, esto es, poseer una serie de aptitudes psicológicas -cognitivas, volitivas y afectivas- que le permiten conocer, valorar y gestionar adecuadamente la información anterior, tomar una decisión y expresarla.

En nuestro país, a medida que se vaya instalando poco a poco este modelo de relación, irá surgiendo poco a poco la preocupación entre los clínicos por la forma de evaluar esta capacidad. Y cada vez será más frecuente que reclamen de psiquiatras y psicólogos ayuda para hacer esta tarea. Por eso es importante que estos profesionales tengan una idea clara sobre los conceptos y herramientas existentes al respecto. Como todos los juicios clínicos, el juicio sobre la capacidad de un paciente siempre será probabilístico y prudencial, no de certeza científica. Por eso, ninguno de los guiones, herramientas o protocolos de evaluación de la capacidad podrá ser nunca un «Santo Grial», el remedio mágico que dará respuesta a todas las dudas y aplacará definitivamente la angustia. Al utilizarlos habrá que asumir la posibilidad de equivocarse, científica, técnica y éticamente. Ello no es óbice para que deban buscarse herramientas de evaluación de la capacidad lo más sensibles 
y específicas posible. El objetivo de este trabajo es precisamente exponer el estado de la cuestión en esta materia.

\section{La cuestión terminológica y conceptual: capacidad, competencia, «capacity», «competency»}

Una primera dificultad es que tanto los textos jurídicos de nuestro país, como la literatura extranjera -norteamericana habitualmente, esto es, estadounidense y canadiense- suelen ser poco claros con los términos y conceptos. Si se lee literatura norteamericana sobre este tema se encontrará con frecuencia con que se usan dos términos distintos: «competency» $\mathrm{y}$ «capacity». «Competency» es un término jurídico, y significa el reconocimiento legal de las aptitudes psicológicas para tomar determinadas decisiones. El término equivalente en nuestro Derecho sería «capacidad de derecho»o «legal». En cambio «capacity» es un término psicológico y clínico. Define las aptitudes psicológicas necesarias para tomar, aquí y ahora, una determinada decisión. Es la que evalúan los médicos, psiquiatras y psicólogos; también los notarios. El término equivalente en nuestro país sería «capacidad de hecho»o «natural», y que con frecuencia, sobre todo en ambientes bioéticos, se ha denominado «competencia». Es importante señalar, por tanto, que la traducción correcta debería ser «cruzada»: «competency» debería traducirse por «capacidad», y «capacity» por «competencia».

En cualquier caso, lo que sucede es que luego en la literatura anglosajona con mucha frecuencia ambos términos se usan indistintamente. Y por lo que respecta a nuestro país, y a pesar de que la ley catalana que regula el consentimiento informado en dicha Comunidad Autónoma (Ley 21/2000) lo utiliza, parece razonable tratar de huir del término «competencia». El motivo es que en nuestro ordenamiento jurídico este término tiene connotaciones que se ajustan mal al contexto de la toma de decisiones por los pacientes. Deberíamos tratar de generalizar el uso del término «capacidad», referido a la «capacidad de obrar», esto es, la que se refiere a las condiciones que permiten a las personas ejercer efectivamente sus derechos y cumplir sus obligaciones. Éstas, como ya hemos visto, son de dos tipos o formas «capacidad (de obrar) legal o de derecho» y «capacidad (de obrar) natural o de hecho». O en su traducción al inglés, «competency» y «capacity» respectivamente.

En realidad ambas están estrechamente relacionadas. Son como las dos caras de una misma moneda. No es posible hablar de una sin referirse a la otra. La capacidad natural o de hecho se refiere a las aptitudes psicológicas internas que permiten a una persona gobernar efectivamente su vida, de tal modo que podamos 
concluir que sus decisiones son auténticas, es decir, que reflejan adecuadamente su propia personalidad, su autonomía moral. Lo que sucede es que esta capacidad de hecho tiene siempre un correlato jurídico que llamamos «capacidad de obrar de derecho o legal». Si cuestionamos la capacidad de hecho de una persona mayor esto tiene automáticamente consecuencias en la esfera de su capacidad de derecho: le retiramos la potestad jurídica para que ejerza activamente y por sí mismo sus derechos y obligaciones, y buscamos a otra persona, su representante, para que lo haga en su lugar.

La relación entre ambas es compleja, y queda ilustrada por la Figura 1. Lo importante es saber si esa limitación de la capacidad es potencialmente transitoria

Figura 1

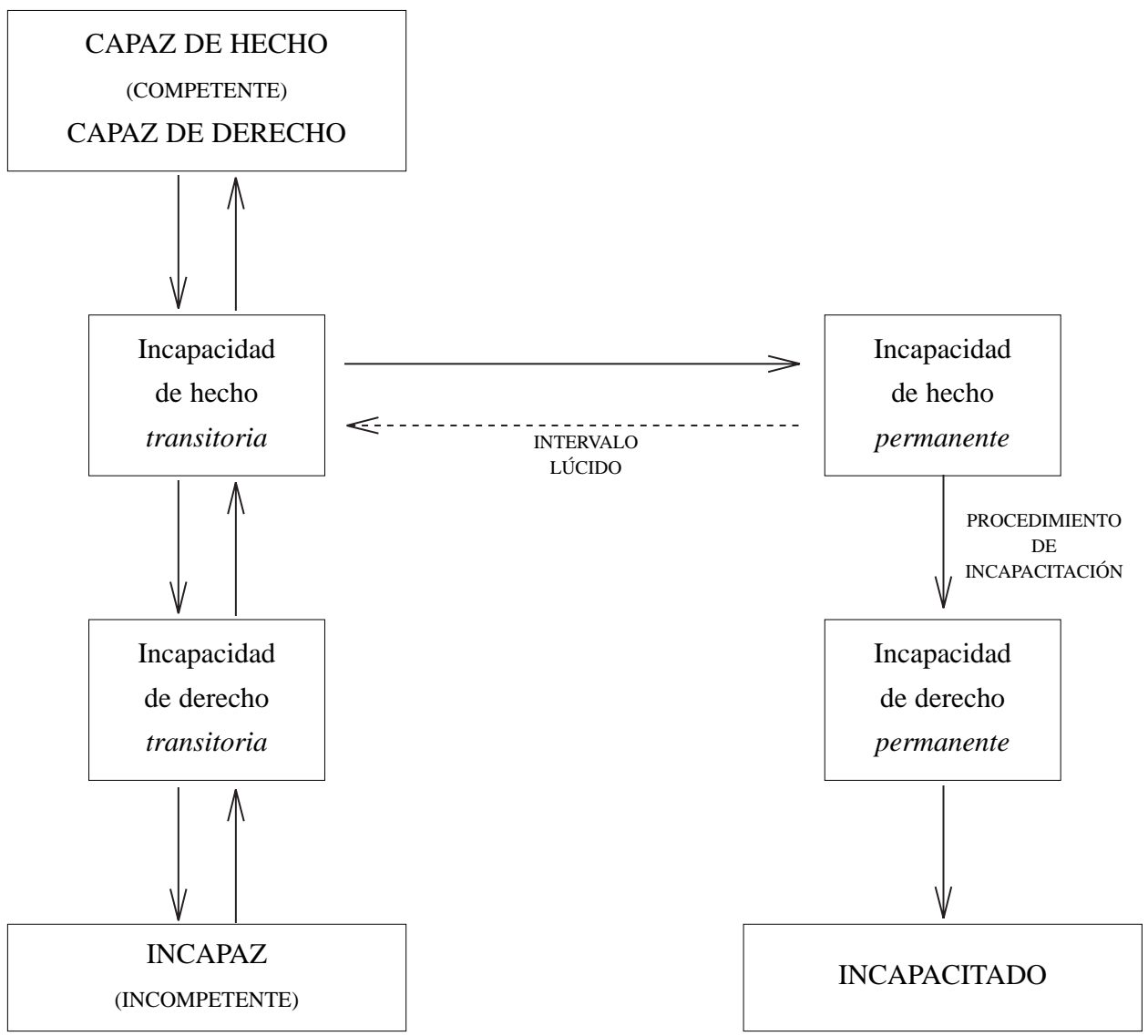


o permanente. En el contexto clínico más habitual lo más frecuente es que hablemos de situaciones potencialmente transitorias. Pero obviamente en determinados campos clínicos, como la psiquiatría o la geriatría, la incapacidad puede devenir permanente.

Para diferenciar mejor a efectos prácticos ambas situaciones, tiende a recurrirse a los términos en negativo. Así, se habla de: «Incapaz», el que no tiene aquí y ahora capacidad de hecho o natural, y como consecuencia de ello pierde transitoriamente su capacidad de derecho. Por ejemplo, un paciente en delirium tremens, en encefalopatía hipercápnica severa $\mathrm{o}$, simplemente, dormido con anestesia general. «Incapacitado», el que no tiene de forma permanente capacidad de derecho o legal, porque un juez ha emitido una sentencia de incapacitación que así lo establece, por padecer una alteración mental que disminuye su juicio, su capacidad de hecho, de forma habitual y no meramente circunstancial. Por ejemplo, un paciente en estado vegetativo permanente, un psicótico con gran deterioro o una demencia avanzada.

La Ley 41/2002 recoge esta distinción en el apartado 3 de su artículo 9. Lo que dice el artículo es que el consentimiento informado debe ser un «consentimiento por representación» en tres supuestos: paciente «incapaz», paciente «incapacitado» y paciente «menor». Este apartado, por tanto, añade a los de «incapaz» e «incapacitado», otros dos conceptos que también hay que conocer y manejar. Uno es el de «consentimiento por representación» que es el tipo de consentimiento que debe emitirse cuando alguien no está en condiciones de emitirlo por él mismo, es decir, no tiene, dicho de forma general «capacidad». En tales casos, no es que el clínico pueda actuar sin dar información o pedir el consentimiento, lo que pasa es que, además de contar en lo posible con el paciente, hay otra persona implicada en la toma de decisiones: el representante. Esto es importante señalarlo porque, con frecuencia, los clínicos tienden a pensar que, cuando un paciente es incapaz, entonces son ellos los que deben retomar el control de la situación y decidir unilateralmente.

El otro concepto importante es el de «menor». La cuestión del consentimiento informado en el caso de los menores es quizás una de las cuestiones más complejas y debatidas en la actualidad, y también una de las que más angustian a los clínicos (3). En este trabajo no abordaremos la cuestión del menor de forma explícita.

Una cosa importante a tener en cuenta es que la capacidad (natural) de los pacientes -al menos de los mayores de edad-debe presumirse siempre, salvo que exista, lógicamente una sentencia de incapacitación. Por eso, de un paciente siempre hay que demostrar la incapacidad, no la capacidad, que se le presume siempre. En caso de duda, la presunción de capacidad debe prevalecer. 
ORIGINALES Y REVISIONES

2. ¿Quién puede y debe evaluar la presunta incapacidad de los pacientes?

Los médicos han realizado evaluaciones de la capacidad de sus pacientes desde siempre. Sin embargo hay que decir que algo tan serio, por las repercusiones jurídicas que tenía, cual es la simultánea limitación y protección de los derechos del paciente, no ha tenido sustrato jurídico en que apoyarse hasta la Ley 41/2002, en concreto los artículos 5.3 y 9.3.a. Los notarios, en cambio, han tenido explícitamente señalada esta potestad y responsabilidad en el Código civil desde hace lustros.

Es importante señalar que la Ley 41/2002 sólo otorga esta función a los «médicos», y no a otro tipo de profesionales sanitarios, como enfermería o psicólogos clínicos. Y la potestad y responsabilidad es «del médico que asiste al paciente» o de su «médico responsable». Ciertamente, antes de decidir la posible incapacidad de un paciente, un médico puede pedir que sea evaluado por otro especialista, médico o no. Pero la responsabilidad última es de él, y no puede delegarla en «el psiquiatra»o «el psicólogo». Estos especialistas tienen sólo una función pericial.

\section{3. ¿Cuándo hay que evaluar la presunta incapacidad de un paciente?}

El hecho de que un médico piense en la conveniencia de evaluar la capacidad de un paciente, para tratar de detectar posibles áreas de incapacidad, no indica necesariamente una actitud paternalista. Más bien suele expresar una actitud de responsabilidad y respeto hacia su paciente. Existen al menos cuatro situaciones en las que al médico deberían disparársele las alarmas ante la posibilidad de que su paciente sea incapaz: 1. El paciente ha tenido un cambio brusco en su estado mental habitual. Estos cambios pueden deberse a problemas psiquiátricos o a alteraciones físicas como hipoxia, infecciones, medicaciones, trastornos metabólicos, etc. 2. El paciente rechaza un tratamiento que está claramente indicado en su caso y no consigue argumentar los motivos con claridad o se basan en ideas y presupuestos excesivamente irracionales. 3. El paciente acepta con facilidad que se le realicen procedimientos muy invasivos, molestos o peligrosos, aparentemente sin ponderar riesgos ni beneficios. 4. El paciente tiene un trastorno neurológico o psiquiátrico de base, previamente conocido, que puede producirle estados transitorios de incapacidad.

\section{4. ¿Cómo se evalúa la presunta incapacidad de un paciente?}

Cuando un clínico se pregunta por la manera de evaluar la capacidad de un paciente, instintivamente vuelve su cabeza hacia el Derecho para ver qué dice al respecto. La desagradable sorpresa es que el Derecho no dice nada concreto. A 
lo sumo dice que un paciente es capaz para dar el consentimiento informado cuando tiene «entendimiento»y «voluntad» suficientes. Pero el ordenamiento no establece ningún criterio objetivo ni ninguna forma práctica de medición de tales cosas.

Cuando, por ejemplo, un juez quiere aclarar tales extremos en el caso de un sujeto, llama a expertos para que hagan un peritaje. Suelen ser médicos forenses, o psiquiatras forenses. Lo sorprendente es que estos especialistas tampoco tienen ningún procedimiento estandarizado para evaluar tales cosas, al menos uno que sea aplicable en un contexto clínico de toma de decisiones. Sus evaluaciones de la capacidad suelen ser el resultado de una combinación de herramientas diagnósticas -test, escalas, etc.-, habitualmente diseñadas para otras funciones, con una evaluación clínica subjetiva mediante exploración y entrevista. Por su parte, los notarios, que también pueden y deben, según el Código civil, hacer tales valoraciones, tampoco tienen procedimiento estandarizado alguno para ello.

Los clínicos españoles, que llevan décadas haciendo «evaluaciones» de facto de la capacidad de los pacientes, tampoco poseen herramientas desarrolladas específicamente para tal función. Lo hacen utilizando una mezcla de sentido común y experiencia profesional que, si bien sirve para salir indemne del trance, no puede sostenerse mucho tiempo más.

La única posibilidad es mirar lo que otros han hecho y plantearse la necesaria validación rigurosa, conceptual y transcultural, de las herramientas por ellos desarrolladas. Además lo ideal sería que, una vez validadas, fueran incorporadas a los programas informáticos de gestión de la historia clínica. Ello favorecería tanto su realización como su registro adecuado.

En Marzo de 1977, tres autores de la Universidad de Pittsburgh (PensilvaniaEE.UU.) y del «Western Psychiatric Institute and Clinic» de la misma ciudad, publicaron en el American Journal of Psychiatry el que puede considerarse el primer trabajo explícitamente dedicado al problema de la evaluación de la capacidad. En dicho trabajo revisaban los tests de capacidad que a su juicio se utilizaban en la práctica habitual, tanto del mundo judicial como del ámbito clínico -sobre todo psiquiátrico- (4). Lo que ellos llamaban «tests» no eran sino lo que hoy llamamos criterios para evaluar la capacidad, cuya determinación es una cuestión que todavía sigue abierta (TABLA 1). 
Tabla 1

Los tests de capacidad según Roth, Meisel y Lidz (1977)

\section{TESTS DE CAPACIDAD \\ (Roth, Meisel y Lidz - 1977)}

1. El paciente es capaz de expresar una elección.

Este test plantea unas exigencias mínimas para que un paciente sea declarado capaz. Éste tan solo tiene que manifestarse a favor o en contra del procedimiento diagnóstico o terapéutico propuesto. No se hace por tanto una valoración de la calidad de la decisión del paciente en ningún sentido, bastando sólo con un «sí», un «no» o un simple «escoja Ud., doctor».

2. El paciente toma una decisión «razonable».

Según este test el paciente es capaz si toma una decisión que pueda ser considerada «correcta»o «responsable». Ésta es la que tomaría una persona «razonable» si se encontrara en la misma posición que el paciente. El énfasis se pone por tanto en el resultado del proceso de toma de decisiones por parte del paciente, más que en el mero hecho de si la decisión existe o en la forma en que ésta se alcanzó.

3. El paciente toma una decisión basada en motivos «racionales».

Este test, a diferencia del anterior, lo que trata de evaluar es la «calidad» del proceso de razonamiento del paciente más que el «resultado» del mismo. Pretende así detectar aquellas decisiones que pudieran ser debidas a una enfermedad mental que afectara al raciocinio del sujeto.

4. El paciente comprende los riesgos, beneficios y alternativas del tratamiento (incluyendo el no-tratamiento).

Según este test el proceso de toma de decisiones de un paciente no necesita ni ser necesariamente «racional» ni producir un resultado «razonable». Pero lo que sí resulta imprescindible, para que el paciente pueda ser considerado capaz, es un grado suficiente de comprensión de la información necesaria para tomar esa decisión, y ello aun cuando el paciente otorgue a cada aspecto de esa información un valor distinto al que le concede el sanitario.

5. El paciente comprende realmente todos los aspectos relevantes de la decisión a tomar y emite un consentimiento verdaderamente voluntario e informado.

Este es un test más complejo, que sólo establece la capacidad o no del sujeto analizando a posteriori cómo el paciente ha comprendido la situación en que se encuentra, la información relevante y cómo ha evaluado estos datos para tomar finalmente una decisión. Se trata de un test exigente, que obliga al sanitario a realizar un minucioso control del proceso de toma de decisiones.

[Tomado de Roth, L. H.; MeISEl, A.; LiDZ, C. W., «Tests of Competency to Consent to Treatment», Am. J. Psychiatry, 1977, 134 (3), pp. 279-284]. 
Estos tests han sido muy conocidos y citados. Pero lo que quizás se ha señalado menos es que en ese trabajo ya se ponía sobre la mesa una cuestión que también ha seguido siendo motivo de debate hasta hoy. A saber, el papel que juega el tipo de decisión clínica en la evaluación de la capacidad y en el establecimiento de los estándares necesarios de cumplimiento de esos criterios. Así, Loren, Roth y Meisel señalaban que, desde su punto de vista, eran sobre todo dos los factores estrechamente relacionados con estas determinaciones: el balance riesgo / beneficio del tratamiento propuesto y el sentido de la decisión del paciente -es decir si se trata de «consentir» o de «rechazar» la medida-.

Para expresar esta interdependencia usaban una tabla de «2x2» (TABLA 2). Esta tabla trataba de expresar que, en las decisiones de consentimiento, el test de capacidad que tiende a utilizar el sanitario es tanto más flexible cuanto menor es el balance riesgo/beneficio. Por el contrario, en las decisiones de rechazo de estas medidas de bajo balance riesgo/beneficio, este test tiende a ser elevado. Y viceversa. Obviamente aquí está la semilla de lo que luego conoceremos como Escala Móvil de Capacidad.

Tabla 2

Factores que, según Roth, Meisel y Lidz, influyen en la selección de un test de capacidad.

BALANCE RIESGO/BENEFICIO

\begin{tabular}{ccc}
\hline DECISIÓN & FAVORABLE & NO - FAVORABLE \\
\hline \multirow{2}{*}{ CONSENTIMIENTO } & Test de Capacidad & Test de Capacidad \\
& BAJO & ALTO \\
\hline \multirow{2}{*}{ RECHAZO } & Test de Capacidad & Test de Capacidad \\
& ALTO & BAJO \\
\hline
\end{tabular}

[Tomado de Roth, L. H.; Meisel, A.; LiDZ, C. W., «Tests of Competency to Consent to Treatment», Am. J. Psychiatry, 1977, 134 (3), pp. 279-284.]

Así pues, este primer trabajo marcaba ya las tres cuestiones básicas en torno a las que ha girado toda la discusión posterior acerca de la evaluación de la capacidad: 1. La determinación de las áreas mentales que deberían evaluarse (criterios). 2. El grado de exigencia que deberían satisfacer dichos criterios (estándares) $\mathrm{y}$, sobre todo, si dichos estándares varían o no en función de la complejidad de la decisión clínica que deba tomarse (Escala Móvil de capacidad). 3. El diseño y validación de protocolos o herramientas estandarizadas de evaluación que puedan aplicarse en la práctica. 
ORIGINALES Y REVISIONES

\subsection{Los criterios de evaluación de la capacidad}

Como se ha visto, la primera revisión acerca de los criterios de evaluación de la capacidad tiene lugar en el marco de la Psiquiatría norteamericana, más en concreto de la Psiquiatría forense. Este marco será el predominante durante toda la década de los ochenta, hasta que comiencen a añadirse al análisis del problema las voces de los neurólogos.

\subsubsection{La perspectiva psiquiátrica}

En los años siguientes a la publicación del estudio de 1977 de Roth, Meisel y Lidz, otro compañero de los anteriores en el «Western Psychiatric Institute and Clinic» de Pittsburgh comenzó a interesarse por la problemática de la capacidad y del consentimiento informado, sobre todo en el campo de la enfermedad mental. Se trataba del psiquiatra Paul S. Appelbaum. En 1979 Appelbaum realizó un primer trabajo sobre los problemas de la evaluación de la capacidad de los pacientes psiquiátricos para consentir ingresos hospitalarios voluntarios (5). A este primer trabajo de tipo teórico le siguió en 1981 un estudio empírico sobre esta cuestión (6). Este segundo estudio es interesante porque constituye una de las primeras aproximaciones a los problemas prácticos de la determinación de la capacidad. La preocupación por las dificultades prácticas de la evaluación de la capacidad en la atención sanitaria llevaron a Appelbaum a publicar, también en 1981, un trabajo sobre esta cuestión junto con Loren H. Roth (7). El trabajo se dirigía sobre todo a los psiquiatras, advirtiendo ya, proféticamente, que la implicación de estos especialistas en la evaluación de la capacidad de todo tipo de pacientes a petición de sus médicos sería una cosa muy frecuente en los años siguientes, con su consiguiente repercusión legal. Pero la aportación más interesante a la cuestión de los criterios y estándares de la evaluación de la capacidad la realizaron Appelbaum y Roth al año siguiente, en 1982. Se trataba de un trabajo sobre la capacidad de los sujetos de experimentación (8).

En este texto Appelbaum y Roth comenzaban constatando cómo la ética de la investigación con seres humanos no había sido capaz hasta la fecha de contestar a la pregunta por la capacidad de los sujetos participantes. Ni siquiera la National Commission, dedicada monográficamente a los problemas éticos de la investigación, había realizado propuestas significativas en este sentido. Todo ello les urgía a realizar una proposición que, si bien orientada a la experimentación en su planteamiento, lógicamente era extensible a la asistencia sanitaria.

Appelbaum y Roth empezaban sugiriendo que los criterios de capacidad se agruparan en cuatro clases, que se encuentran en la TABLA 3, cuyo nivel de exi- 
ORIGINALES Y REVISIONES

Tabla 3

Los 4 criterios de evaluación de la capacidad de PS Appelbaum y Loren Roth (1982)

\begin{tabular}{|c|c|c|}
\hline $\begin{array}{c}\text { CRITERIOS } \\
\text { DE CAPACIDAD }\end{array}$ & $\begin{array}{c}\text { INDICIOS } \\
\text { CARACTERÍSTICOS }\end{array}$ & $\begin{array}{c}\text { ASPECTOS PSIQUIÁTRICOS } \\
\text { RELEVANTES }\end{array}$ \\
\hline $\begin{array}{l}\text { El sujeto expresa } \\
\text { una decisión. } \\
\text { (Expressing a choice) }\end{array}$ & $\begin{array}{l}\text { - Manifiesta su consentimien- } \\
\text { to. } \\
\text { - Expresa positivamente inte- } \\
\text { rés en tomar parte en el } \\
\text { estudio. } \\
\text { - Coopera adecuadamente. } \\
\text { - Responde a las preguntas. }\end{array}$ & $\begin{array}{l}\text { - Mutismo: Estupor catatóni- } \\
\text { co o depresión severa. } \\
\text { - Manía o Agitación catatóni- } \\
\text { ca. } \\
\text { - Desórdenes psicóticos del } \\
\text { pensamiento. } \\
\text { - Marcada ambivalencia: es- } \\
\text { quizofrenia, estados obsesi- } \\
\text { vos. }\end{array}$ \\
\hline $\begin{array}{l}\text { 2. El sujeto comprende } \\
\text { realmente los problemas } \\
\text { más relevantes del estudio. } \\
\text { (Understanding) }\end{array}$ & $\begin{array}{l}\text { - Tiene suficiente capacidad } \\
\text { cognitiva. } \\
\text { - Comprende la naturaleza, } \\
\text { riesgos y beneficios del pro- } \\
\text { cedimiento. } \\
\text { - Conoce las alternativas, con } \\
\text { sus ventajas e inconvenientes. } \\
\text { - Sabe que tiene que tomar } \\
\text { una decisión. } \\
\text { - Sabe quién es, dónde está, } \\
\text { qué está leyendo al leer el for- } \\
\text { mulario de consentimiento y } \\
\text { qué significa firmarlo. } \\
\text { - Conoce las consecuencias } \\
\text { tanto de su participación } \\
\text { como de su negativa. }\end{array}$ & $\begin{array}{l}\text { - Grado de inteligencia. Coe- } \\
\text { ficiente de inteligencia (retra- } \\
\text { so mental, alteraciones orgá- } \\
\text { nicas) y capacidad para desa- } \\
\text { rrollar una vida normal (en- } \\
\text { fermedad mental crónica). } \\
\text { - Habilidades lingüísticas. } \\
\text { - Grado de atención y de } \\
\text { orientación. } \\
\text { - Capacidades memorísticas. } \\
\text { - Repercusiones de daño ce- } \\
\text { rebral y de intoxicaciones. }\end{array}$ \\
\hline $\begin{array}{l}\text { 3. El sujeto maneja } \\
\text { racionalmente la } \\
\text { información de } \\
\text { forma adecuada. } \\
\text { (Reasoning) }\end{array}$ & $\begin{array}{l}\text { - Tiene buen juicio. } \\
\text { - Es racional y evalúa correc- } \\
\text { tamente la realidad. } \\
\text { - Puede tomar decisiones de } \\
\text { manera adecuada. }\end{array}$ & $\begin{array}{l}\text { - Ilusiones y alucinaciones. } \\
\text { - Alteraciones del curso del } \\
\text { pensamiento. } \\
\text { - Ansiedad, euforia, agitación. } \\
\text { - Fobias extremas, pánico. } \\
\text { - Preocupaciones obsesivas. } \\
\text { - Pasividad y dependencia } \\
\text { extremas. }\end{array}$ \\
\hline
\end{tabular}


Tabla 3

Los 4 criterios de evaluación de la capacidad de PS Appelbaum y Loren Roth (1982)

(Continuación)

\begin{tabular}{|c|c|c|}
\hline $\begin{array}{c}\text { CRITERIOS } \\
\text { DE CAPACIDAD }\end{array}$ & $\begin{array}{c}\text { INDICIOS } \\
\text { CARACTERÍSTICOS }\end{array}$ & $\begin{array}{c}\text { ASPECTOS PSIQUIÁTRICOS } \\
\text { RELEVANTES }\end{array}$ \\
\hline $\begin{array}{l}\text { 4. El sujeto aprecia } \\
\text { correctamente la } \\
\text { situación en la que } \\
\text { se encuentra. } \\
\text { (Appreciation) }\end{array}$ & $\begin{array}{l}\text { - Es consciente, tanto cogniti- } \\
\text { va como afectivamente, de } \\
\text { la situación. } \\
\text { - Es consciente de las conse- } \\
\text { cuencias de su consenti- } \\
\text { miento o su rechazo. } \\
\text { - Sabe quién es y los motivos } \\
\text { por los que consiente. } \\
\text { - Reconoce, de forma madu- } \\
\text { ra, las implicaciones de las } \\
\text { alternativas de acción. } \\
\text { - Evalúa adecuadamente lo } \\
\text { que es relevante para for- } \\
\text { marse un juicio acerca de la } \\
\text { cuestión. } \\
\text { - Para todo lo anterior, es } \\
\text { capaz de darse cuenta de: } \\
\text { que tiene un problema que } \\
\text { se adapta a las exigencias } \\
\text { del estudio. } \\
\text { que el estudio tiene objeti- } \\
\text { vos investigadores y no sólo } \\
\text { terapéuticos. } \\
\text { que en su cuidado pueden } \\
\text { participar simultáneamente } \\
\text { investigadores y no-investi- } \\
\text { gadores. } \\
\text { que el tratamiento puede ser } \\
\text { randomizado, a doble ciego, } \\
\text { con placebo, etc. }\end{array}$ & $\begin{array}{l}\text { - Negaciones acerca de: } \\
\text { de la existencia de su enfer- } \\
\text { medad o de la gravedad de } \\
\text { la misma. } \\
\text { de la condición investigado- } \\
\text { ra del estudio. } \\
\text { de la posibilidad de mejora } \\
\text { con y sin la participación en } \\
\text { el estudio. } \\
\text { de la metodología del estu- } \\
\text { dio. } \\
\text { - Capacidad para el pensa- } \\
\text { miento abstracto afectada } \\
\text { por: } \\
\text { Bajo Coeficiente } \\
\text { Inteligencia. } \\
\text { Bajo nivel educativo. } \\
\text { Psicosis. } \\
\text { Daño cerebral orgánico. } \\
\text { - Alteraciones psicóticas: } \\
\text { Distorsiones. } \\
\text { Proyecciones. } \\
\text { Nihilismo. } \\
\text { Desesperanza-abandono. }\end{array}$ \\
\hline
\end{tabular}

[Tomado de Appelbaum, P. S., Roth, L. H., «Competency to Consent to Research: a Psychiatric Overview». Arch. Gen. Psychiatry, 1982, 39, pp. 951-8].

gencia es progresivamente mayor. Como puede verse los autores suprimían el segundo criterio propuesto por Roth, Meisel y Lidz, el relativo a «lo razonable» de la decisión tomada por el paciente. Pero además introducían dos innovaciones en la explicación de cada uno de ellos: a) sugerían una serie de «aspectos psiquiátri- 
cos relevantes» que podían interferir o influir en cada caso en la satisfacción del criterio en cuestión y b) detectaban los problemas de la evaluación empírica e incluso esbozaban, muy someramente, sugerencias sobre cómo explorar a los pacientes en un subapartado de cada criterio denominado «evaluando la capacidad».

La trascendencia de este trabajo ha sido enorme. De hecho, aunque posteriormente otros autores hayan realizado otras aportaciones, estos cuatro criterios han quedado como los esenciales, y han comenzado a usarse para construir algoritmos sencillos de toma de decisiones (9). De hecho, estos 4 criterios constituyen la base de uno de los procedimientos clínicos de evaluación de la capacidad más sólidamente validado en el momento actual, el MacArthur Competence Assessment Tool, diseñado por Thomas Grisso y Paul S Appelbaum (TABLA 4). El primer esbozo se encuentra en un artículo suyo del New England Journal of Medicine de 1988, en el que retomaban estos 4 criterios como los esenciales para cualquier evaluación de la capacidad (10).

Tabla 4

Criterios y puntuaciones del MacCAT-T

\begin{tabular}{clcc}
\hline CRITERIO & \multicolumn{1}{c}{ SUBCRITERIO } & PP & PT \\
\hline Comprensión & Comprensión de la enfermedad & $0-2$ & $0-6$ \\
& Comprensión del tratamiento & $0-2$ & \\
& Compresión de Riesgos y Beneficios & $0-2$ & \\
\hline \multirow{2}{*}{ Apreciación } & De la situación de la enfermedad & $0-2$ & $0-4$ \\
& Del objetivo general del tratamiento & $0-2$ & \\
\hline \multirow{2}{*}{ Razonamiento } & Lógico-secuencial & $0-2$ & $0-8$ \\
& Lógico-comparativo & $0-2$ & \\
& Predictivo (consecuencias derivadas & & \\
& de las posibles elecciones) & $0-2$ & \\
& Consistencia interna del & & \\
& Procedimiento de elección & $0-2$ & \\
\hline Elección & Expresa una decisión & $0-2$ & $0-2$ \\
\hline
\end{tabular}

PP: Puntuación Parcial de cada subcriterio.

PT: Puntuación Total posible del criterio.

\subsubsection{La perspectiva neurológica}

A finales de los años ochenta comenzaron a oírse voces que señalaban que las discusiones sobre capacidad se habían centrado en el análisis de los elementos psicológicos de la toma de decisiones, pero habían dejado de lado el estudio del sustrato biológico de esos elementos. Según esta idea los déficits de carácter neu- 
rológico que alteren las actividades mentales pueden suscitar la incapacidad de un paciente para tomar decisiones de una manera tanto o más importantes que las alteraciones puramente psíquicas -si es que existen con independencia de las anteriores-. Dicho de otra manera, según estos autores, si el psiquiatra tiene mucho que hacer y decir en una evaluación de la capacidad, el neurólogo no tiene precisamente menos. Para Michael P. Alexander -uno de los primeros en situarse en esta perspectiva- la capacidad de un sujeto depende de que realicen adecuadamente tres pasos antes de tomar una decisión (11).

El primero consiste en reconocer que lo que se le pide es que tome una decisión intencionadamente. Para ello el sujeto tiene que tener intactos a) los mecanismos neocorticales que le permiten mantener la comunicación con el exterior mediante el procesamiento de información compleja, y b) el sistema límbico que le permitirá añadir el tinte volitivo y emocional básico a la elaboración de sus respuestas.

El segundo estriba en poder activar todos los mecanismos neurosensoriales que le permitan procesar información y emitir respuestas. Estos mecanismos son fundamentalmente cuatro: a) revisión de experiencias pasadas semejantes; b) obtención de información nueva; c) procesamiento aritmético, visual, emocional, etc., de toda la información anterior; d) mantenimiento de expectativas acerca del resultado de la decisión y de sus posibles consecuencias emocionales.

El tercer paso es la realización práctica de la resolución tomada, que puede ser externa (una acción o una decisión concreta) o interna (reevaluación del proceso y reinicio del mismo). Según Alexander para poder realizar adecuadamente estos tres pasos el sujeto tiene que mantener en un grado suficiente las 7 actividades mentales siguientes: 1) Atención; 2) Memoria; 3) Lenguaje; 4) Percepción espacial ; 5) Área de cálculo, comunicación, etc.; 6) Razonamiento; y 7) Actividad emotiva y afectiva.

Un afásico severo, por ejemplo, puede tener una afectación neocortical que genera un déficit de lenguaje que le incapacita para realizar los pasos primero y segundo. Por el contrario, un Síndrome del Lóbulo Frontal presentará a menudo alteraciones de diverso tipo que le incapacitarán para realizar el tercer paso. Pero lo más importante de todo es que, según Alexander, muchos de los enfermos en los que él había evaluado la capacidad y en los que había encontrado déficits lo suficientemente severos como para ser considerados incapaces, habrían sido encontrados aptos con los criterios y estándares que hemos venido describiendo en el epígrafe anterior.

A principios de los noventa, otros tres autores -Freedman, Stuss y Gordoninsistían sobre todo en las actividades cognitivas necesarias para un buen ejercicio de la capacidad, dejando a un margen los componentes afectivos (12). Para ellos 
están actividades son las cuatro que se encuentran en la TABLA 5, y su evaluación correcta debería fijarse en las cinco actividades siguientes: a) la atención; b) la comprensión de información básica relacionada con el tipo de decisión en cuestión; c) la retención de la información; d) la expresión de deseos; e) la apercepción y juicio en relación a las cuestiones que se estén debatiendo.

\section{Tabla 5}

Áreas cognitivas relevantes para una evaluación adecuada de la capacidad de un paciente según Freedman, Stuss y Gordon.

\begin{tabular}{ll}
\hline Atención & \\
\hline \multirow{2}{*}{ Lenguaje } & - Lenguaje espontáneo \\
& - Comprensión de información verbal \\
& - Lectura comprensiva \\
& Escritura \\
\hline \multirow{2}{*}{ Memoria } & - Reciente \\
& - Remota \\
\hline \multirow{2}{*}{ Funciones del Lóbulo Frontal } & - Apercepción (awareness) \\
& - Juicio \\
\hline
\end{tabular}

Pero en realidad, esta aproximación no es muy diferente de los cuatro criterios de Appelbaum y Grisso. Quizás por eso, son los que se han convertido en el punto de referencia obligado.

Lo más interesante de todas estas aportaciones es haber señalado, con razón, que la exploración de la capacidad no puede ser meramente psiquiátrica sino que precisa atender igualmente al área neurológica. Esto era tan evidente que los sucesivos intentos de elaboración de protocolos de evaluación específica de la capacidad o los trabajos sobre la forma en que se realizan dichas evaluaciones en la clínica diaria van a contrastar sus resultados con tests -como el Minimental de Folstein- que exploran esta área.

De hecho, el único intento español de elaborar un procedimiento de evaluación de la capacidad, el «Documento Sitges», del que se hablará más adelante, hace un abordaje eminentemente neurológico de la cuestión, quizás porque el tipo de pacientes a los que va dirigido, los pacientes con demencia, así lo exige.

\subsection{Los estándares de capacidad y la discusión de la «Escala Móvil de Capacidad»}

El debate en la literatura norteamericana acerca del grado de cumplimiento de los diferentes criterios de capacidad que debían satisfacer las personas para ser 
consideradas capaces o incapaces ha estado dominado por el debate sobre el concepto de «Escala Móvil de Capacidad». Esta idea ha sido defendida y desarrollada por James Drane (13), y por Allen Buchanan y Dan Brock (14-15).

Para los defensores del concepto de «Escala Móvil» la capacidad es algo relacionado directamente con la decisión clínica concreta que deba tomarse. Las decisiones clínicas complejas, que impliquen un balance riesgo beneficio complejo, tales como rechazar la realización de una radiografía de tórax para estudiar una hemoptisis en un paciente fumador, requerirán grados de capacidad más elevados. Sin embargo, las decisiones clínicas sencillas, tales como aceptar la realización de análisis de sangre para hacer el seguimiento de un diabético estable y no complicado, precisaran grados de capacidad más bajos.

El concepto de Escala Móvil parte, por tanto, de la idea de que el establecimiento de la capacidad implica el establecer un punto o nivel de corte en una línea continua que va desde la incapacidad total a la capacidad total. Pues bien, la teoría de la Escala Móvil, lo que dice es que dicho punto de corte no es fijo, sino móvil, y que se desplaza en función de la complejidad de las decisiones a tomar. La capacidad no es pues «simétrica», sino «asimétrica». De hecho Drane habla de tres niveles o grados de dificultad de las decisiones. En el Nivel I, el de menor dificultad, la mayor parte de las personas serán consideradas capaces, mientras que en el Nivel III, que implica alta complejidad de las decisiones, muchas personas serán consideradas incapaces.

Esta propuesta ha generado un debate importante, aunque en general de perfil más académico que clínico $(16 ; 17 ; 18)$. El principal argumento en contra es que, de alguna forma, reintroduce un condicionante de tipo paternalista al ejercicio de la autonomía de los sujetos, pues la valoración acerca de lo que sea una decisión «difícil» o no depende de un balance riesgo-beneficio que realizan otras personas diferentes al propio sujeto, habitualmente los médicos (19).

\subsection{Las herramientas de evaluación de la capacidad en el contexto clínico}

\subsubsection{La situación actual en el mundo anglosajón}

Si la década de los ochenta se dedicó a discutir los criterios y estándares de evaluación de la capacidad, en la década de los noventa cobraron protagonismo decisivo el diseño de herramientas o protocolos clínicos de evaluación de la capacidad. Lo que parece dominar la presente década es una prolongación de la anterior, pero ahora el énfasis se pone en la investigación comparativa entre los diferentes protocolos de evaluación, a la búsqueda del instrumento más rápido, sencillo y fiable. Ya en otro lugar se han expuesto algunos de los protocolos pues- 
tos en marcha en los primeros años de la década de los ochenta (20). En lo que sigue se expondrán las líneas principales de desarrollo que ha experimentado la bibliografía en el resto de los noventa y hasta la actualidad.

Lo primero que hay que señalar es que la investigación en este campo ha estado vinculada fundamentalmente a tres áreas de la Medicina, la Psiquiatría/Psicología, la Geriatría, y, actuando como campo intermedio entre ambas, la Neurología. Las actividades sanitarias en el marco de las cuales se ha producido esta investigación sobre evaluación de la capacidad para decidir han sido dos: las decisiones clínicas (diagnósticas y terapéuticas) y la participación en proyectos de investigación (21-22). Los pacientes con los que se ha investigado más han sido los mayores, en concreto los pacientes con demencia (23), y los enfermos psiquiátricos, en concreto los esquizofrénicos (24) y, en menor medida, los pacientes con depresión (25).

Hay desarrollos importantes en otro ámbito diferente, aunque relacionado estrechamente con los anteriores: el ámbito forense. El objetivo ha sido desarrollar procedimientos para evaluar la capacidad tanto en procesos del ámbito civil como del penal. Los primeros suelen implicar procedimientos de incapacitación, o, simplemente, la evaluación de la capacidad de testigos para participar en juicios, los segundos, determinaciones de la imputabilidad del acusado.

La evaluación práctica de la capacidad ha utilizado tres tipos de herramientas que, según su grado decreciente de especificidad son las siguientes:

1. Test neuropsicológicos, como el Minimental, el Weschler, etc. El problema de estos tests es que no son herramientas diseñadas específicamente para esta tarea. Tienen un papel muy importante en la investigación sobre evaluación de la capacidad y, seguramente, un hueco como sistemas complementarios de evaluación en la práctica.

2. Protocolos generales de evaluación de la capacidad, de los que hablaremos más adelante.

3. Protocolos específicos de evaluación de capacidad. Evalúan la capacidad en relación con tareas o procedimientos concretos. Por ejemplo, la «Hopkins Competency Assessment Tool» (HCAT) (26) y las «Health Care Proxy Guidelines» (HCP) (27) evalúan fundamentalmente la capacidad para completar una voluntad anticipada del tipo «Poder de representación» («Durable Power of Attorney») (28). El «Competence Interview Schedule» (CIS) evalúa la capacidad para consentir o rechazar la terapia electroconvulsiva (29). Y la «Evaluation to Sign Consent» (30) (ESC) y la «California Scale of Appreciation» (31) (CSA) la capacidad para consentir la participación en un proyecto de investigación. 
Los más interesantes son sin duda los protocolos generales. Estos pueden adscribirse a alguna de las 3 familias siguientes (32-33):

1. Guiones de entrevista: Son simples listados de preguntas que se sugieren a los clínicos como las más adecuadas para hacer una exploración de la capacidad durante una entrevista clínica (34). No dan indicaciones sobre la forma de evaluar las preguntas.

2. «Tests» o Protocolos: Consisten en guiones estructurados o semiestructurados de entrevista que añaden un sistema de evaluación de las respuestas, habitualmente mediante puntuaciones numéricas.

3. Protocolos con viñetas: Son protocolos que, en vez de explorar la capacidad en relación con la decisión real y concreta a tomar, como suelen hacer los anteriores, utilizan escenarios hipotéticos.

Los más desarrollados son los protocolos que incorporan sistemas de puntuación, utilicen o no escenarios hipotéticos para la evaluación. En la TABLA 6 se encuentran los 6 protocolos que pueden considerarse más importantes en el momento presente.

Tabla 6

Los protocolos generales de evaluación de la capacidad más importantes

\begin{tabular}{|c|c|c|c|c|c|c|}
\hline DENOMINACIÓN & ACRÓNIMO & AUTOR & PAÍS & AÑO & TIPO & PUNTÚA \\
\hline Aid to Capacity Evaluation & ACE & Etchells y otros $(50)$ & Canadá & 1999 & Entrevista semiestructurada & SÍ \\
\hline Capacity Assessment Tool & CAT & Carney y otros (51) & EE.UU. & 2001 & Entrevista estructurada & Sí \\
\hline $\begin{array}{l}\text { Capacity to Consent } \\
\text { to Treatment Instrument }\end{array}$ & CCTI & Marson y otros (52) & EE.UU. & 1995 & 2 escenarios y entrevista & SÍ \\
\hline $\begin{array}{l}\text { Decision Assessment } \\
\text { Meausure }\end{array}$ & DAM & Wong y otros (53) & UK & 2000 & Entrevista semiestructurada & SÍ \\
\hline $\begin{array}{l}\text { Hopemont Capacity } \\
\text { Assessment Interview }\end{array}$ & $\mathrm{HCAI}$ & Edelstein (54) & EE.UU. & 1999 & 2 escenarios y entrevista & Sí \\
\hline $\begin{array}{l}\text { MacArthur Competence } \\
\text { Assessment Tool }\end{array}$ & $\begin{array}{l}\text { MacCAT-T* } \\
\text { MacCAT-CR } \\
\text { MacCAT-CA }\end{array}$ & $\begin{array}{l}\text { Grisso \& } \\
\text { Appelbaum } \\
(55-56)\end{array}$ & EE.UU. & 1998 & $\begin{array}{l}\text { Entrevista semiestructurada } \\
\text { (1 escenario en la version CR) }\end{array}$ & SÍ \\
\hline
\end{tabular}

* El McCAT existe en tres versiones «T» (Treatment) para decisiones clínicas de tratamiento, «CR» (Clinical Research), para participar en proyectos de investigación, $\mathrm{y}$ «CA» (Criminal Adjudication), para imputabilidad.

Quizás las dos mejores revisiones que existen actualmente en la literatura sobre las luces y sombras de los protocolos de evaluación de la capacidad son la de Edward Sturman (35) y la de Jennifer Moye, y otros (36). De sus análisis puede concluirse que el estado de la cuestión es el siguiente: 
1. Los déficits en los criterios de comprensión (understanding) y razonamiento (reasoning) detectados por los protocolos se correlacionan bien con los déficits detectados por los tests clínicos de evaluación neuropsicológica habitualmente utilizados. La relación es más débil en el caso del criterio de elección (expressing a choice), y sobre todo, en el de apreciación (appreciation). Seguramente esto último se debe a que el grado de abstracción y personalización requerido por este criterio no es adecuadamente evaluado por los tests neuropsicológicos. La «California Scale of Appreciation» (CSA) fue diseñada expresamente para evaluar esta cuestión en el caso de la participación en proyectos de investigación.

2. Existe correlación entre los tests de detección de deterioro cognitivo, como el Minimental, y los tests de evaluación de la capacidad, sobre todo en relación con el criterio de comprensión, pero hace falta investigar más consistencia de esa relación.

3. Se ha detectado correlación entre los resultados de los test de capacidad y otras variables, como por ejemplo, la experiencia previa con procesos de toma de decisiones sanitarias similares, el grado de socialización (37) o el estatus socioeconómico. El tipo de enfermedad también parece ser una variable importante que condiciona los resultados (38). En el caso de los pacientes psicóticos la severidad de los síntomas negativos parece relacionarse con la disminución de la capacidad, pero no la de los síntomas positivos o depresivos (39). Recientemente se ha puesto de manifiesto que un breve entrenamiento previo destinado a mejorar las habilidades de los pacientes esquizofrénicos, antes de evaluarlos con un test de capacidad, mejora los resultados del mismo (40).

4. Existen estudios de fiabilidad de los resultados de estos protocolos, pero son insuficientes. Las propiedades psicométricas no son bien conocidas (41). Los estudios de concordancia entre los diferentes test o protocolos no son concluyentes. En general la concordancia en los resultados en el criterio de comprensión es mejor que en los demás. El de menor concordancia es el de apreciación (42). No hay buenos estudios de fiabilidad test-retest, sobre todo que evalúen sujetos sanos, que no están sometidos en principio a las posibles fluctuaciones de la capacidad derivadas de una situación de enfermedad. Se necesitan más estudios de concordancia entre observadores, porque aunque los resultados parecen ser buenos, a veces hay resultados contradictorios (43-44).

5. La investigación sobre la validez de los protocolos se enfrenta a un problema metodológico difícil de resolver: la ausencia de un «patrón oro» que permita estimar la sensibilidad y especificidad de los tests. El más utilizado es la evaluación realizada por clínicos entrenados y experimentados, como forenses, psiquiatras o geriatras. 
6. Los tamaños muestrales utilizados en la mayor parte de los estudios son pequeños, lo que limita la validez de los resultados. De todas formas la complejidad del constructo implica diseños metodológicos con pruebas múltiples, para los que es difícil reclutar muchos sujetos.

Estos 6 puntos hacen difícil que pueda elevarse un protocolo de evaluación de la capacidad por encima de otro de manera absoluta. Con todo, la tendencia que puede percibirse en la literatura es considerar el McCAT como el protocolo de referencia, tanto en la toma de decisiones clínicas (McCAT-T) como de investigación (McCAT-CR) (45). Pero sin duda alguna, hace falta más investigación complementaria que profundice en los aspectos antes señalados, y dé respuesta a los nuevos interrogantes que van apareciendo en el horizonte (46): Por ejemplo, ¿pueden diseñarse protocolos que sean más breves, fáciles y rápidos de aplicar que los actuales? Si se acepta que el «patrón oro» sean las evaluaciones realizadas por expertos, ¿qué requisitos deben cumplir tales expertos para ser considerados tales? (47) ¿Cómo dar respuesta al problema ético que plantea la posible incapacidad de los sujetos de investigación para consentir a participar en proyectos de investigación sobre protocolos de evaluación de la capacidad? (48).

\subsubsection{La situación actual en España}

En España, salvo error u omisión, no existen protocolos validados de evaluación de la capacidad. Lo más cercano a ello es el «Documento Sitges» (49). Se trata de un estudio realizado con metodología de consenso Delphi por un grupo multidisciplinar de expertos en el amplio campo de las demencias (geriatras, neurologos, trabajadores sociales, psicólogos, abogados, etc.). El objetivo del estudio era establecer los criterios básicos que orienten la evaluación de la capacidad de las personas con demencia para tomar decisiones en seis ámbitos diferentes: Decisiones que influyen en la propia salud (S), sobre la participación en investigaciones (I), relacionadas con el patrimonio (P), relativas a actividades propias no laborales (A), decisiones sobre sí mismo diferentes de las anteriores (O) y, por último, decisiones sobre otras personas sobre las que se tiene responsabilidad no laboral (T). Los 16 criterios seleccionados tienen una orientación muy neurológica (TABLA 7). Cada criterio puede ser evaluado en cada paciente asignándole alguna de las puntuaciones siguientes: 1 Normal; 2 Deterioro ligero; 3 Deterioro moderado; 4 Deterioro intenso; 5 Función abolida. Los estándares se fijan mediante una doble consideración. Por una parte se discriminan en cada ámbito de decisión dos niveles de dificultad según el grado de complejidad de la decisión a tomar: «De grado mayor» $(\mathrm{M}) \mathrm{y}$ «de grado menor» $(\mathrm{m})$. Por otra parte, mediante 
procedimiento Delphi, se asignan las puntuaciones entre 1 y 5 que, en cada criterio y cada tipo de decisión, se considera la alteración máxima permisible.

\section{Tabla 7}

Aspectos neurológicos intervienen en la toma de decisiones y que configuran criterios de capacidad, según el «Documento Sitges»

Aspectos neurológicos que intervienen en la toma de decisiones

1. Nivel de conciencia (Nc)

2. Percepción de estímulos (Pc)

3. Atención (At)

4. Gnosias $(\mathrm{Gn})$

5. Lenguaje: comprensión (L-C)

6. Lenguaje: expresión (L-E)

7. Cálculo $(\mathrm{Cc})$

8. Memoria episódica de hechos recientes (Me)

9. Memoria episódica autobiográfica (Ma)

10. Orientación espacial (Oe)

11. Praxia constructiva (P-C)

12. Funciones ejecutivas $(\mathrm{Fe})$

13. Razonamiento abstracto (Ra)

14. Motivación-autocontrol-introspección (MAI)

15. Ideación espontánea (Ie)

16. Afectividad-estado emocional (AE)

La evaluación práctica de la capacidad se basa, por tanto, en la evaluación neuropsicológica realizada mediante los tests habitualmente utilizados en el estudio del paciente con demencia. Los autores han realizado el interesantísimo esfuerzo de tabular qué criterios de la lista de los 16 propuestos pueden ser explorados con cada test o escala. El más rentable es la batería CAMDEX, pues es capaz de evaluar 15 de los 16 criterios propuestos.

Las luces y oscuridades del «Documento Sitges» son evidentes. De hecho, los mismos autores las señalan:

«Debe quedar claro que los resultados son arbitrarios en la medida en que se derivan de la opinión subjetiva de personas, aunque éstas sean expertas y hayan alcanzado consenso. Sería necesario trasladar las apreciaciones a la práctica, en una muestra controlada de individuos, aplicando métodos objetivos que permitan evaluar la validez de las tablas y determinar la manera en que deben ser ajustadas. En todo caso, esperamos que la tarea realizada será útil para afrontar esta investigación y contribuya a la defensa del derecho a decidir, que resulta imprescindible para participar en la actividad social». 
ORIGINALES Y REVISIONES

\section{Conclusión: el largo camino que queda por hacer}

La conclusión última que puede deducirse de todo lo visto hasta el momento es que la evaluación de la capacidad de los pacientes para tomar decisiones sigue siendo un área donde existen más preguntas que respuestas. Y si esto es cierto en el caso de los países anglosajones, en el caso de nuestro país la situación es realmente preocupante. Es imprescindible y urgente que se realicen estudios de validación rigurosos que permitan poner a disposición de los clínicos e investigadores españoles herramientas que permitan evaluar la capacidad de los pacientes y sujetos de investigación. A este respecto, a modo de conclusión, pueden efectuarse 10 sugerencias prácticas:

1. Que se efectúe en nuestro país una validación transcultural rigurosa del MacCAT-T y del MacCAT-CR. Esto implica aceptar como válidos los criterios de capacidad de comprensión, apreciación, razonamiento y elección.

2. Que esa validación se haga con tamaños de muestras suficientes para tener potencia estadística.

3. Que en esa validación se comparen sujetos sanos con sujetos enfermos, en concreto, al menos con pacientes con demencia, pacientes esquizofrénicos y pacientes con alguna enfermedad crónica. También debería diferenciarse la situación intrahospitalaria de la extrahospitalaria.

4. Que en esa validación se comparen los resultados del MacCAT con los resultados obtenidos por otros test psiquiátricos o neuropsicológicos. En pacientes mayores deberían usarse, para comparar, el Minimental. Y en pacientes con demencia, el CAMDEX.

5. Que en dicha validación se utilicen como «patrones oro», clínicos con entrenamiento explícito en la evaluación de la capacidad. Para evitar la sobrecarga de los pacientes o sujetos de experimentación, esa evaluación puede hacer mediante la valoración de los videos grabados de las entrevistas en las que se apliquen el MacCAT y los tests neuropsicológicos.

6. Es preciso incluir medidas de la fiabilidad, test-retest y concordancia entre observadores, así como de las propiedades psicométricas del MacCAT.

7. Es preciso garantizar la corrección ética de todo el proyecto de investigación, y solucionar el problema de la obtención del consentimiento informado.

8. Más adelante convendría extender la investigación sobre herramientas de evaluación de la capacidad a los menores de edad, un área que en los próximos años se revelará como crecientemente conflictiva.

9. Otra área que debería ser rápidamente desarrollada, una vez tengamos un protocolo estándar validado como el MacCAT, es la investigación acerca de versiones abreviadas de las herramientas, que permitan evaluaciones más rápidas pero igualmente fiables. 
10. Nada de lo anterior será posible si no se mejora la formación general de los clínicos y los juristas en torno a la evaluación de la capacidad en el contexto clínico. La mención expresa de los juristas es fruto de la constatación personal reiterada de que, si bien los clínicos españoles tienen, en general, un desconocimiento importante y preocupante de esta cuestión, el nivel de los juristas en general no es mucho mejor. Y este es un área donde el trabajo conjunto de ambos es imprescindible.

\section{BIBLIOGRAFÍA}

(1) Faden, R. R.; Beauchamp, T. L., A History and a Theory of Informed Consent, Nueva York, Oxford Univ. Press, 1986.

(2) Simón, P., «Diez mitos en torno al consentimiento informado», An. Sist. Sanit. Navar., 2006, 29 (supl. 3), pp. 29-40.

(3) Gracia, D.; Jarabo, Y.; Martín Espíldora, N.; Ríos, J., «Toma de decisiones en el paciente menor de edad», Med. Clin., Barcelona, 2001, 117, pp. 179-90.

(4) Roth, L. H.; Meisel, A.; LiDz, C. W., «Tests of Competency to Consent to Treatment», Am. J. Psychiatry, 1977, 134, pp. 279-284.

(5) Appelbaum, P. S.; Bateman, A. L., «Competency to Consent to Voluntary Psychiatric Hospitalization: A Theoretical Approach», Bull. Am. J. Psychiatry Law, 1979, 7, pp. 390-9.

(6) Appelbaum, P. S.; Mirkin, S. A.; Bateman, A. L., «Empirical Assessment of Competency to Consent to Psychiatric Hospitalization», Am. J. Psychiatry, 1981, 138 (9), pp. 1170-6.

(7) Appelbaum, P. S.; Roth, L. H., «Clinical Issues in the Assessment of Competency», Am. J. Psychiatry, 1981, 138 (11), pp. 1462-7. En Julio de 1982 apareció en la misma revista un caso clínico comentado donde insistían de nuevo en esta problemática. Rотн, L., y otros, «The Dilemma of Denial in the Assessment of Competency to Refuse Treatment», Am. J. Psychiatry, 1982, 139 (7), pp. 910-913.

(8) Appelbaum, P. S.; Roth, L. H., «Competency to Consent to Research: a Psychiatric Overview», Arch. Gen. Psychiatry, 1982, 39, pp. 951-8.

(9) Miller, S. S.; MARIN, D. B., «Assessing Capacity», Psychiatric Emergencies, 200, 18 (2), pp. 233-242.

(10) Appelbaum, P. S.; Grisso, T., «Assessing Patient's Capacities to Consent to Treatment», NEJM, 1988, 319 (25), pp. 1635-8.

(11) AleXander, M. P., «Clinical Determination of Mental Competence», Arch. Neurol., 1988, 45, pp. 23-6.

(12) Freedman, M.; Stuss, D. T.; Gordon, M., «Assessment of Competency: the Role of Neurobehavioral Deficits», Ann. Intern. Med., 1991, 115, pp. 203-8.

(13) Drane, J. F., «The Many Faces of Competency», Hastings Cent. Rep., 1985, abril, pp. 17-21. Las múltiples caras de la competencia, en CouCEIRO, A., Madrid, Triacastela, 1999, pp. 163-176.

(14) Buchanan, A.; Brock, D. W., Deciding for Others. The Ethics of Surrogate Decisionmaking, Nueva York, Oxford University Press, 1989.

(15) Buchanan, A., «Mental Capacity, Legal Competence and Consent to Treatment», J. $R$. Soc. Med., 2004, 97, pp. 415-20.

(16) WILKS, I., «The Debate Over Risk-Related Standards of Competence», Bioethics, 1997, 11 , pp. 419-20.

(17) Cale, G. S., «Risk-Related Standards of Competence: Continuing the Debate Over Riskrelated Standards of Competence», Bioethics, 1999, 13, pp. 131-48. 
(18) WiLKs, I., «Asymmetrical Competence», Bioethics, 1999, 13, pp. 154-9.

(19) Demarco, J. P., «Competence and Paternalism», Bioethics, 2002, 16 (3), pp. 231-245.

(20) Simón, P., El consentimiento informado, Madrid, Triacastela, 2000, pp. 302-309.

(21) Moser, D. J., y otros, «Capacity to Provide Informed Consent for Participation in Schizophrenia and HIV Research», Am. J. Psychiatry, 2002, 159 (7), pp. 1201-7.

(22) Pinals, D. A.; ApPelbaum, P. S., «The History and Current Status of Competence and Informed Consent in Psychiatric Research», Isr. J. Psychiatry Relat. Sci., 2000, 31 (2), pp. 82-94.

(23) KIM, S. Y. H., y otros, «Assessing the Competence of Persons with Alzheimer's Disease in Providing Informed Consent for Participation in Research», Am. J. Psychiatry, 2001, 158, pp. 712-7.

(24) Jeste, D. V.; Depp, C. A.; Palmer, B. W., «Magnitude of Impairment in Decisional Capacity in People with Schizophrenia Compared to Normal Subjects: An Overview», Schizophrenia Bull., 2006, 32 (1), pp. 121-8.

(25) Appelbaum, P. S., «Competence of Depressed Patients for Consent to Research», Am. J. Psychiatry, 1999, 156 (9), pp. 1380-4.

(26) Janofsky, J. S.; McCarthy, J. S.; Folstein, M. F., «The Hopkins Competency Assessment Test: A Brief Method for Evaluating Patient's Capacity to Give Informed Consent», Hosp. Community Psychiatry, 199, 43 (2), pp. 132-6.

(27) MezeY, M., y otros, «Decision-making Capacity to Execute a Health Care Proxy: Development and Testing Guidelines», JAGS, 2000, 48, pp. 179-187.

(28) Sin embargo, el HCAT ha comenzado a ser utilizado recientemente como evaluador indirecto general de la capacidad. LuCHA, P. A.; KroPCHO, L.; FrANCIS, M., «Acute Pain and Narcotic Use Does Not Impair the Ability to Provide Informed Consent: Evaluation of a Competency Assessment Tool in the Acute Pain Patient», American Surgeon, 2006, 72 (2), pp. 154-7.

(29) BEAN, G., y otros, «The Assessment of Competence to Make a Treatment Decision: An Empirical Approach», Canadian Journal of Psychiatry, 1996, 41, pp. 85-92.

(30) Deronzo, E. G.; Conley, R. R.; Love, R., «Assessment of Capacity to Give Consent to Research Participation: State of the Art and Beyond», J. Health Care Law Policy, 1998, 1, pp. 6687. El formulario está disponible, por ejemplo, en http://www.dhmh.state.md.us/oig/irb/pdf/attachment6-2006.pdf (visitada el 14-XI-2006).

(31) SAKs, E. R., «The California Scale of Appreciation: A New Instrument to Measure the Appreciation Component of Capacity to Consent to Research», American Journal of Geriatric Psychiatry, 2002, 10, pp. 166-174.

(32) Sullivan, K., «Neuropsychological Assessment of Mental Capacity», Neuropsychology Review, 2004, 14 (3), pp. 131-142.

(33) TunZI, M., «Can the Patient Decide? Evaluating Patient Capacity in Practice», American Family Physician, 2001, 64 (2) pp. 299-306.

(34) Finucane, P.; Myser, C.; Ticehurst, S., «Is she Fit to Sign, Doctor? Practical Ethical Issues in Assessing the Competence of Elderly Patients», Med. J. Aust., 1993, 159, pp. 400-3.

(35) Sturman, E. D., «The Capacity to Consent to Treatment and Research: A Review of Standardized Assessment Tools», Clinical Psychology Review, 2005, 25, pp. 954-74.

(36) MoYE, J., y otros, «Empirical Advances in the Assessment of the Capacity to Consent To Medical Treatment. Clinical Implications and Research Needs», Clinical Psychology Review, 2006, 26, pp. 1054-1077.

(37) Allen, R. S., y otros, «Advance Care Planning in Nursing Homes: Correlates of Capacity and Possession of Advance Directives», Gerontologist, 2003, 43 (3), pp. 309-17.

(38) Palmer, B. W., y otros, «Assessment of Capacity to Consent to Research Among Older Persons with Schizophrenia, Alzheimer Disease, or Diabetes Mellitus: Comparison of a 3-Item Questionnaire with a Comprehensive Standardized Capacity Instrument», Arch. Gen. Psychiatry, 2005, 62 (7), pp. 726-9.

(39) PALMeR, B. W.; Jeste, D. V., «Relationship of Individual Cognitive Abilities to Specific Components of Decisional Capacity Among Middle-aged and Older Patients with Schizophrenia», Schizophrenia Bull., 2006, 32 (1), pp. 98-106. 
(40) Moser, D. J., y otros, «Using a Brief Intervention to Improve Decisional Capacity in Schizofrenia Research», Schizophrenia Bull., 2006, 32 (1), pp. 116-200.

(41) ZAPF, P. A.; RoESCH, R., "An Investigation of the Construct of Competence: A Comparison of the FIT, the MacCat-CA and the MacCat-T», Law and Human Behaviour, 2005, 29 (2), pp. 229-252.

(42) MoYe, J., y otros, «Capacity to Consent to Treatment: Empirical Comparison of Three Instruments in Older Adults with and without Dementia», Gerontologist, 2004, 44 (2), pp. 166-75.

(43) Marson, D. C., y otros, «Consistency of Physician Judgments of Capacity to Consent in Mild Alzheimer's Disease», JAGS, 1997, 45, pp. 453-7.

(44) CAIRns, R., y otros, «Reliability of Mental Capacity Assessments in Psychiatric Patients», Br. J. Psychiatry, 2005, 187, pp. 372-8.

(45) Casarett, D. J., «Assessing Decision-Making Capacity in the Setting of Palliative Care Research», J. Pain. Symptom Management, 2003, 25 (4), pp. 6-13.

(46) Appelbaum, P. S., «Decisional Capacity of Patients with Schizophrenia to Consent to Research»: Taking Stock», Schizophrenia Bull, 2006, 32 (1), pp. 22-25.

(47) KIM, S. Y., «When Does Impairment Become Decisional Incompetence. Ethical and Methodological Issues in Capacity Research in Schizophrenia», Schizophrenia Bull., 2006, 32 (1), pp. 92-97.

(48) SAKs, E. R., y otros, «Meta-consent in Research on Decisional Capacity: A «Catch-22»», Schizophrenia Bull., 2006, 32 (1), pp. 42-46.

(49) BoAda, M.; RoBles, A. (eds), Análisis y reflexiones sobre la capacidad para tomar decisiones durante la evolución de una demencia: «Documento Sitges», Barcelona, Glosa, 2005. Disponible en la web de la Sociedad Española de Neurología. http://www.sen.es/ (visitada el 15-XI2007).

(50) Etchells, E., y otros, Assessment of Patients Capacity to Consent to Treatment. Journal of General Internal Medicine 1999, 14, pp. 27-34. El protocolo está disponible en http://www.utoronto.ca/jcb/disclaimers/ace.htm (visitada el 15-XI-2007).

(51) CARneY, M., y otros, «The Development and Piloting of a Capacity Assessment Tool», The Journal of Clinical Ethics, 2001, 12, pp. 17-23.

(52) MARSON DCCODY, y otros, «Neuropsychological Predictors of Competency in Alzheimer's Disease Using a Rational Reasons Legal Standard», Archives of Neurology, 1995, 52, pp. 955-9.

(53) Wong, J. G., y otros, «The Capacity of People with a Mental Disability to Make a Health Care Decision», Psychological Medicine, 2000, 30, pp. 295-306.

(54) Edelstein, B., Hopemont Capacity Assessment Interview Manual and Scoring Guide, Morgantown, W. V., West Virginia University, 1999.

(55) Grisso, T., Applebaum P. S., Assessing Competence to Consent to Treatment, Nueva York, Oxford University Press, 1998.

(56) Appelbaum, P. S., MacCAT-CR. Mac Arthur Competence Assessment Tool for Clinical Research, Sarasota (FL - USA), Professional Resource Press, 2001.

* Pablo Simón-Lorda. Doctor en Medicina. Especialista en Medicina Familiar y Comunitaria. Magister en Bioética por la UCM. Profesor de la Escuela Andaluza de Salud Pública. Granada. Correspondencia: Pablo Simón-Lorda. Escuela Andaluza de Salud Pública. Apdo. 2070. 18080 Granada. Telf.: 958 027400. Fax: 958 027503. pablo.simon.easp@juntadeandalucia.es

** Fecha de recepción: 7-XII-2007 (aceptado 10-IV-2008). 